\title{
The extended pectoralis major flap for reconstruction of the upper posterior chest wall and axilla
}

\author{
Niklas Iblher, MD, Vincenzo Penna, MD, Arash Momeni, MD, Nestor Torio Padron, MD, and G. Bjoern Stark, MD, Prof, \\ Freiburg, Germany
}

Supplemental material is available online.

$\mathrm{A}$ lthough the pectoralis major muscle as a basis for a variety of flaps is still a workhorse for head and neck reconstruction $^{1}$ and a flap of choice for anterior chest wall reconstruction, ${ }^{2}$ coverage of dorsal upper chest wall defects including the axilla are hardly described. In certain cases when infiltration of a tumor or trauma or previous damage to the vascular supply precludes the use of adjacent muscle flaps, a pectoralis major musculocutaneous island flap can offer a valuable alternative.

\section{Anatomy}

The pectoralis major muscle originates from the medial part of the clavicle, the sternocostal border of the first 6 ribs, and the external oblique muscle aponeurosis.

The main functions of the muscle are adduction and medial rotation of the arm. Sacrifice of this muscle leads to only minimal functional deficit because adjunct muscles of the shoulder belt can almost completely compensate for the loss. ${ }^{3}$

The main vascular supply to the pectoralis muscle and its overlying skin derives from the pectoral branch of the thoracoacromial artery originating from beneath the midportion of the clavicle and coursing toward the xiphoid. Further vascular supply originates from perforating branches of the internal thoracic artery, from perforating vessels that derive from the 5th to 7 th intercostal arteries, and from the lateral thoracic artery.

The skin island should be centered over the pectoralis major muscle but can be considerably extended caudally (up to a size of $45 \times 18 \mathrm{~cm}$ and more) by including the rectus fascia owing to a rich vascular network anastomosing with the superior epigastric system, ${ }^{4}$ as has been shown in injection studies of the thoracodorsal system. $^{5}$

From the Department of Plastic and Hand Surgery, University of Freiburg Medical Center, Freiburg, Germany.

Received for publication Oct 9, 2007; accepted for publication Nov 25, 2007.

Address for reprints: Niklas Iblher, MD, Department of Plastic and Hand Surgery, University of Freiburg Medical Center, Hugstetter Strasse 55, 79106 Freiburg, Germany (E-mail: niklas.iblher@uniklinik-freiburg.de).

J Thorac Cardiovasc Surg 2008;136:790-1

$0022-5223 / \$ 34.00$

Copyright $\odot 2008$ by The American Association for Thoracic Surgery doi:10.1016/j.jtcvs.2007.11.066

\section{Technique}

The estimated defect is marked and an equivalent skin island is designed along the pectoralis major muscle axis (Figure 1). The island can extend well beyond the lower border of the pectoralis muscle so long as a sufficient part of the skin island is localized above the pectoralis major. If a larger transverse dimension of the skin island is required, the nipple may be included in the flap in men.

The patient is positioned in a lateral decubitus position. The flap is raised in a retrograde manner by incising the skin along the preoperative markings down to the anterior rectus sheath caudally. The anterior rectus sheath has to be included in extended flaps. Staying below the abdominal fascia allows a straightforward dissection. The origins of the pectoralis major at the caudal ribs and the sternum in continuation with the fascia are separated, and the subpectoral plane is entered. If possible, the lateral part of the muscle should be spared to prevent loss of the anterior axillary fold. At the cranial edge of the island, the dissection is performed down to the pectoralis major muscle and from there upward toward the clavicle. The vascular pedicle is visualized on the undersurface of the muscle and isolated, leaving a protective cuff of muscle around it. The insertion of the muscle to the humerus lateral to the pedicle can then be separated and the flap transferred to the recipient area (Figure 2). If a transposition into a dorsal defect is planned, a subcutaneous tunnel is made. Skin islands of a dimension up to $45 \times 18 \mathrm{~cm}$ can be safely elevated, still allowing primary wound closure at the donor site (Figure 3).

In men, large skin islands may incorporate the nipple areola complex, which can later be retransplanted as a free skin transplant in a secondary procedure with local anesthesia.

Three representative clinical summaries are presented in an online appendix (E-Appendix).

\section{Discussion}

Numerous articles report algorithms for chest wall reconstruction. Chest wall defects are more common anteriorly because the primary underlying causes are located here (eg, breast cancer, radiation ulcer, and sternotomy wounds) and their management is well documented. Posterior chest wall defects are less common, their approach far less well documented, and thus are left out of suggested algorithms. Among the regional options for coverage are the trapezius flap, the latissimus dorsi flap, flaps of the (para)scapular system, gluteus flaps, or paraspinous muscle flaps. If these local flaps are precluded by the extent of resection or compromised vascular pedicles, free tissue transfer can offer a flexible solution, although the availability of recipient vessels may be difficult in some cases. For defects of the upper posterior chest wall and shoulder, the pectoralis major flap offers a valuable alternative with minimal donor site defect and functional impairment. ${ }^{3}$ This is supported by reports on patients with congenital deficiency of this muscle.

Flap dissection is quick and straightforward. A wide arc of rotation and a large skin island are among the advantages of the 

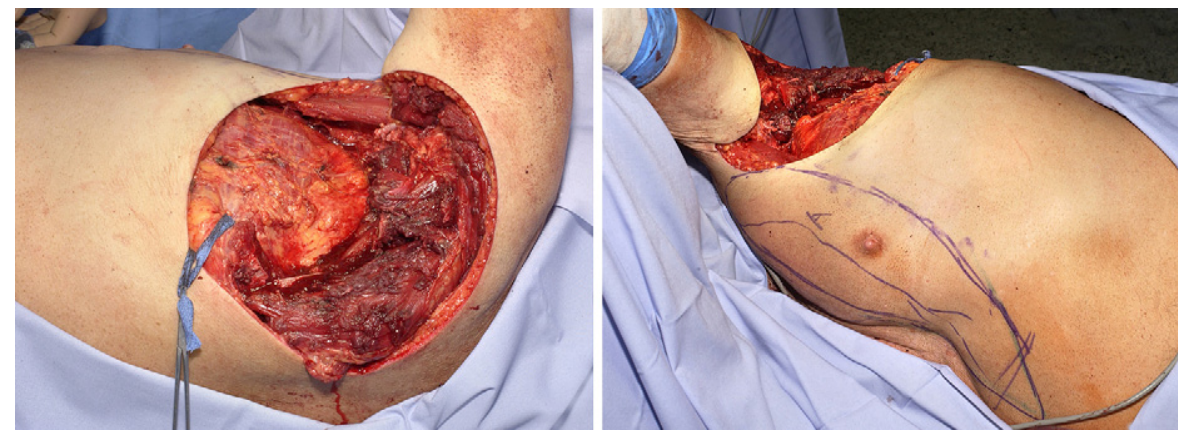

Figure 1. Defect and flap design.
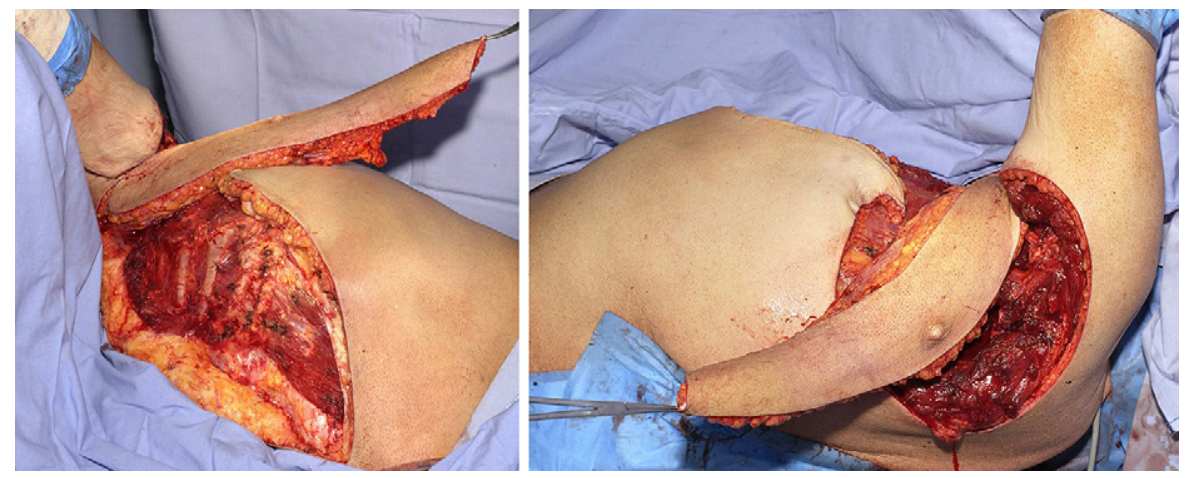

Figure 2. Flap transposition.
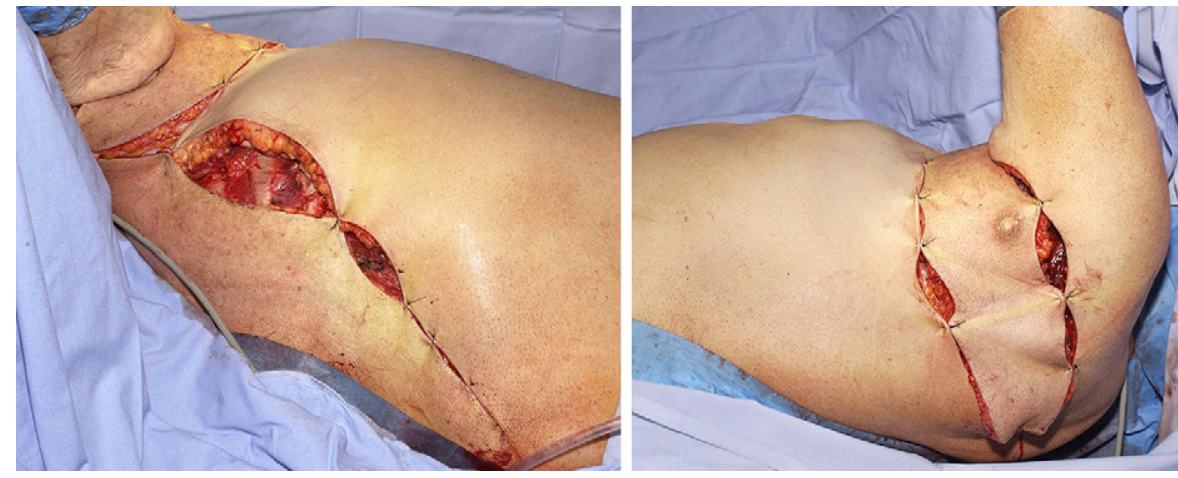

Figure 3. Wound closure.

pectoralis major flap. Owing to the anastomosis of vascular network with the epigastric system, large skin islands that extend far below the pectoralis muscle down to the periumbilical region can be harvested, with primary donor site closure being possible in almost all patients (Figure 3).

The modern armamentarium of plastic surgery offers a wide range of safe reconstructive flap transfers with minor morbidity for chest wall reconstruction. Resectability of solid tumors hardly should have to be excluded on the basis of the impossibility to cover the resulting defect. In patients with an upper dorsal or axillary defect in which use of dorsal muscle flaps is precluded owing to resection, vascular compromise, or other reasons, the extended pectoralis major musculocutaneous flap offers a safe, fast, and reliable alternative.

\section{References}

1. Vartanian JG, Carvalho AL, Carvalho SM, Mizobe L, Magrin J, Kowalski LP. Pectoralis major and other myofascial/myocutaneous flaps in head and neck cancer reconstruction: experience with 437 cases at a single institution. Head Neck. 2004;26:1018-23.

2. Tobin GR. Pectoralis major muscle-musculocutaneous flap for chest-wall reconstruction. Surg Clin North Am. 1989;69:991-1006.

3. Har-El G, Krespi YP, Har-El R. Physical rehabilitation after myocutaneous flaps. Head Neck. 1990;12:218-24.

4. Russell RC, Feller AM, Elliott LF, Kucan JO, Zook EG. The extended pectoralis major myocutaneous flap: uses and indications. Plast Reconstr Surg. 1991;88:814-23.

5. Reid CD, Taylor GI. The vascular territory of the acromiothoracic axis. $\mathrm{Br}$ J Plast Surg. 1984;37:194-212. 


\section{E-Appendix Clinical Summaries}

PATIENT 1. A 24-year-old man with a history of a mole removal 7 months before presentation and a failed reoperation with axilla dissection owing to an increasing mass at the incision site several weeks later was transferred to our department. The histologic workup of the second operation revealed a malignant melanoma with lymphatic metastasis to the axilla. Staging showed no signs of further metastasis. The patient received preoperative chemotherapy. Figure E1A shows the preoperative situation. Wide excision included the margo lateralis, spina, and pars membranacea of the scapula, as well as the periscapular muscles including the teres major and minor, infraspinatus, supraspinatus, and latissimus dorsi, which precluded use of dorsally based muscle flaps. Axillary dissection was performed and R0 status was achieved (Figure E1B).

Defect coverage was achieved with a musculocutaneous pectoralis flap with an abdominally extended skin island (Figure E1C). Primary closure of the donor site was possible. Healing was uneventful and the patient was discharged on postopeerative day 7. Six months after the operation, the patient achieved nearly symmetric active shoulder movement (Figure E1D). Retransplantation of the nipple to its original location was offered to the patient but he declined. Seven months after the operation, a local lymph node metastasis was removed and 20 months after the operation another two suspicious lymph nodes were resected, but did not show any malignant cells. Up to now, 3.5 years after the operation, the patient is free of relapse. The patient reports almost normal shoulder function.

Patient 2. A 76-year-old retired oncology professor had a scapular swelling that he attributed to a recent mosquito bite. Magnetic resonance imaging showed a suspicious fatty soft tissue tumor. Incisional biopsy was performed and revealed a low-grade liposarcoma (Figure E2A). The tumor infiltrated the latissimus and infraspinatus muscles and reached the posteroinferior surface of the scapula. Wide resection included the caudal part of the trapezius, the latissimus dorsi, teres minor and major, infraspinatus, and the periostium of the scapula, which precluded the use of muscle flaps from the dorsal trunk (Figure E2B). An extended pectoralis major musculocutaneous flap was raised, tunneled subcutaneously through the axilla, and placed into the defect. Primary closure of the donor site was possible. Postoperative recovery was uneventful. Figure E2C displays the postoperative result after 3 months. One year after the operation, the patient is free of relapse.

Patient 3. A 66-year-old patient had a growing tumor above the left scapula. Incisional biopsy demonstrated a pleomorphic sarcoma. Wide resection was performed by the senior author (GBS). The latissimus muscle was infiltrated and had to be resected, thereby being excluded for local coverage. Large parts of the infraspinatus fossa were resected, preserving the inferior tip and spine (Figure E3A). Axillary lymph nodes of levels one and two were resected en bloc, including a suspicious pectoralis minor muscle. Because the skin was infiltrated up to the anterior axillary line, a large island flap $(35 \times 15 \mathrm{~cm})$ extending far into the hypogastric region had to be raised (Figure E3B). To assure perfusion of the skin island, the anterior sheath of the rectus muscle was raised with it. The flap was rotated $120^{\circ}$ and completely covered the defect (Figure E3C ). The donor site defect could be closed primarily (Figure E3D). Resection margins were free of tumor and wound healing was uneventful. Again, nipple transplantation was offered to the patient but has not yet been performed. Postoperative radiotherapy was performed after complete wound healing and was well tolerated. Figure E3E shows the result 18 months postoperatively. A mild bulging of the flap is noticeable but does not disturb the patient. Three years postoperatively, there is no recurrence of the sarcoma. 


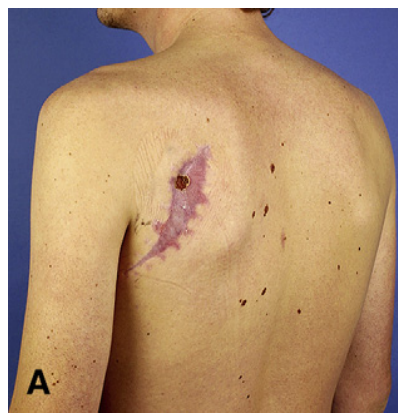

Figure E1A. Patient 1: Preoperative situation with $\mathrm{R} 2$ resection of a malignant melanoma. 


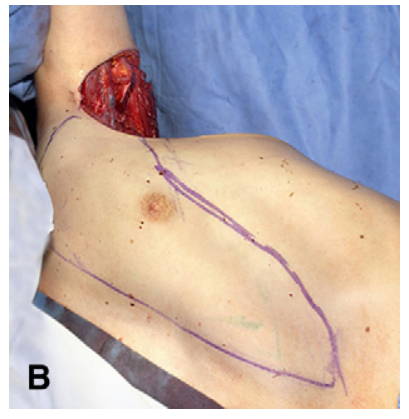

Figure E1B. Patient 1: Design of extended musculocutaneous pectoralis major flap. 


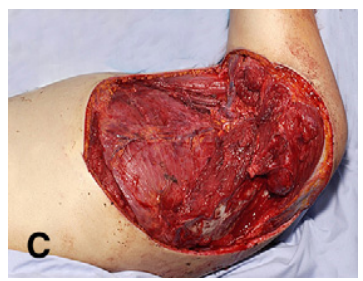

Figure E1C. Patient 1: Intraoperative situation. Above wide resection including the margo lateralis, spina, and pars membranacea of the scapula and the periscapular and parascapular muscles. 

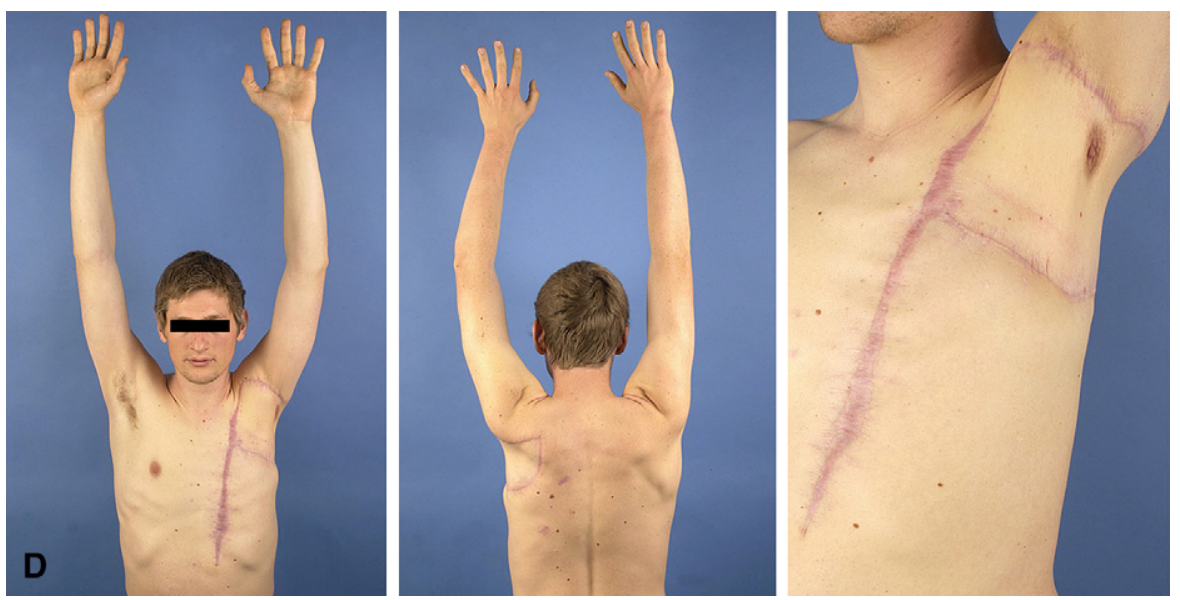

Figure E1D. Patient 1: Six months postoperatively showing almost normal shoulder function. 


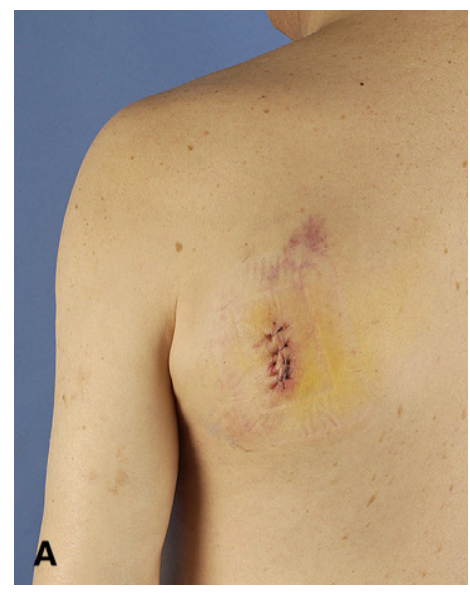

Figure E2A. Patient 2: Situation after incisional biopsy revealing a liposarcoma. 


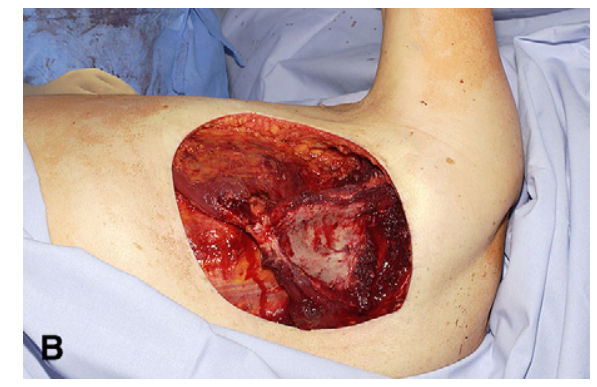

Figure E2B. Patient 2: Defect after wide resection including the lower trapezius, latissimus, teres major and minor, and the periostium of the lower scapula. Insertion of the serratus muscle was free of tumor and was left intact. 

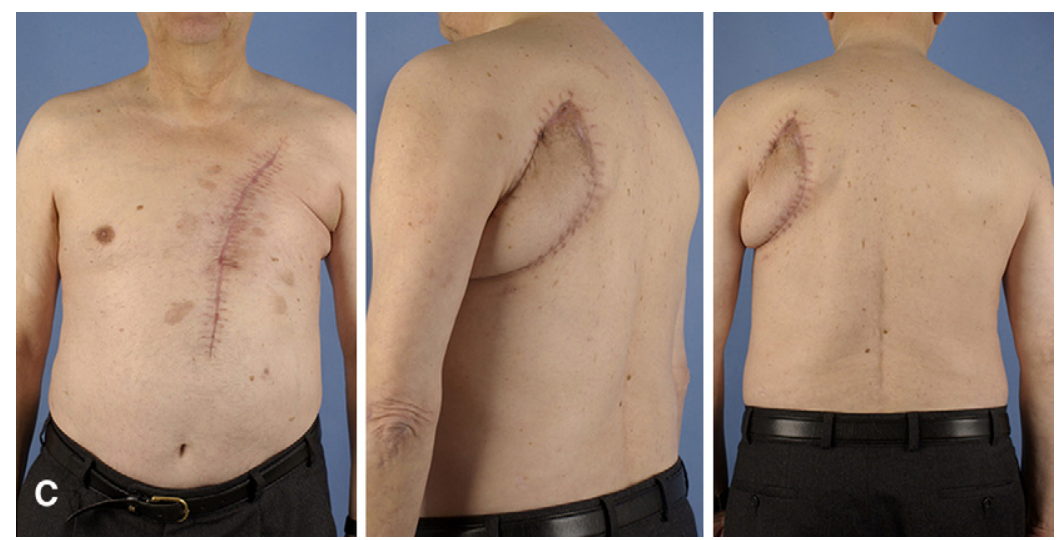

Figure E2C. Patient 2: Three months postoperatively. 


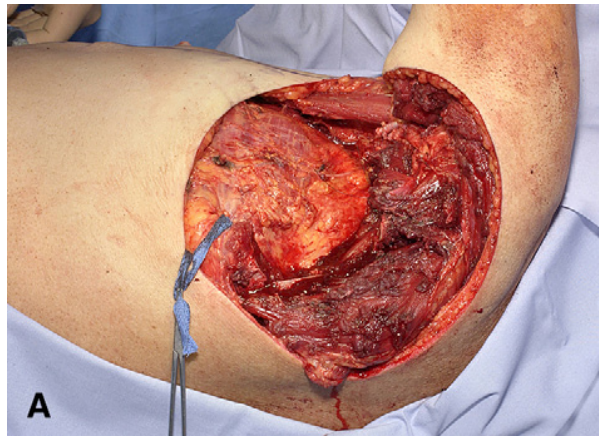

Figure E3A. Patient 3: Defect after wide resection of a pleomorphic sarcoma. 


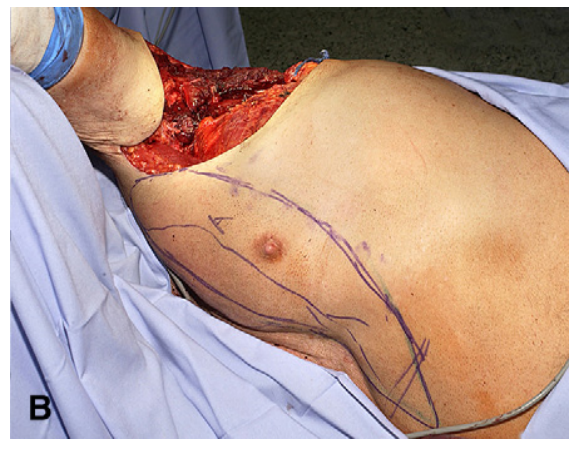

Figure E3B. Patient 3: Flap design of extended pectoralis major myocutaneous flap. 

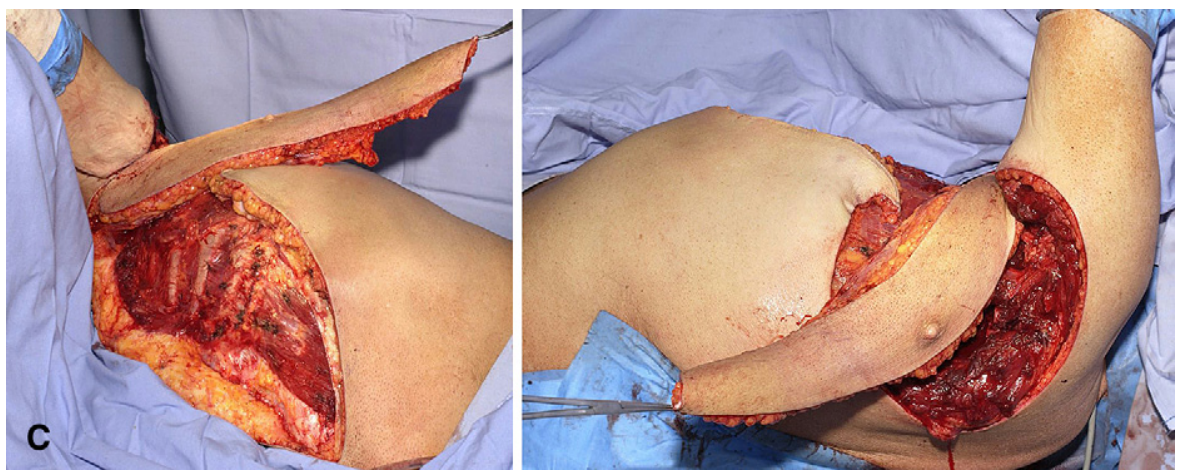

Figure E3C. Patient 3: Flap transposition. 

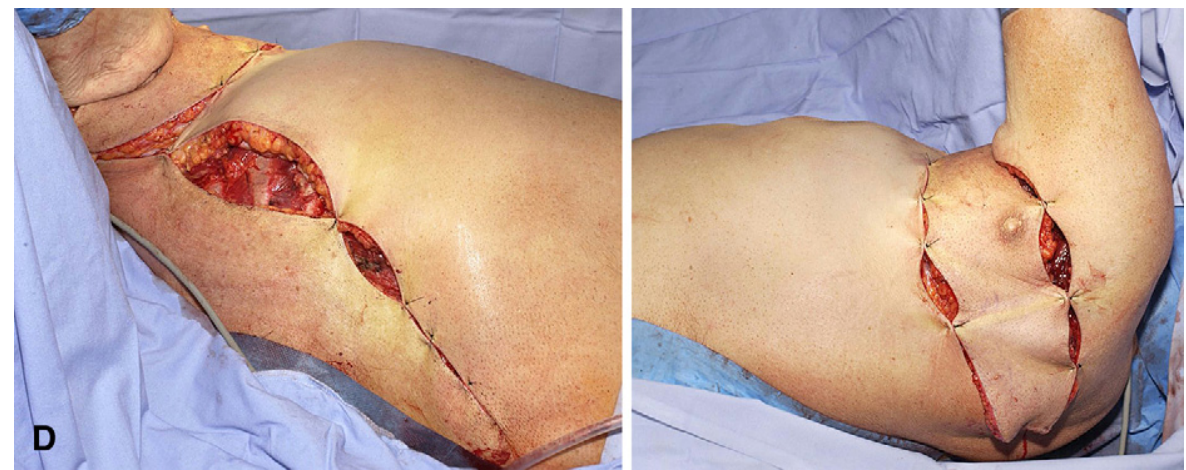

Figure E3D. Patient 3: Wound closure. 

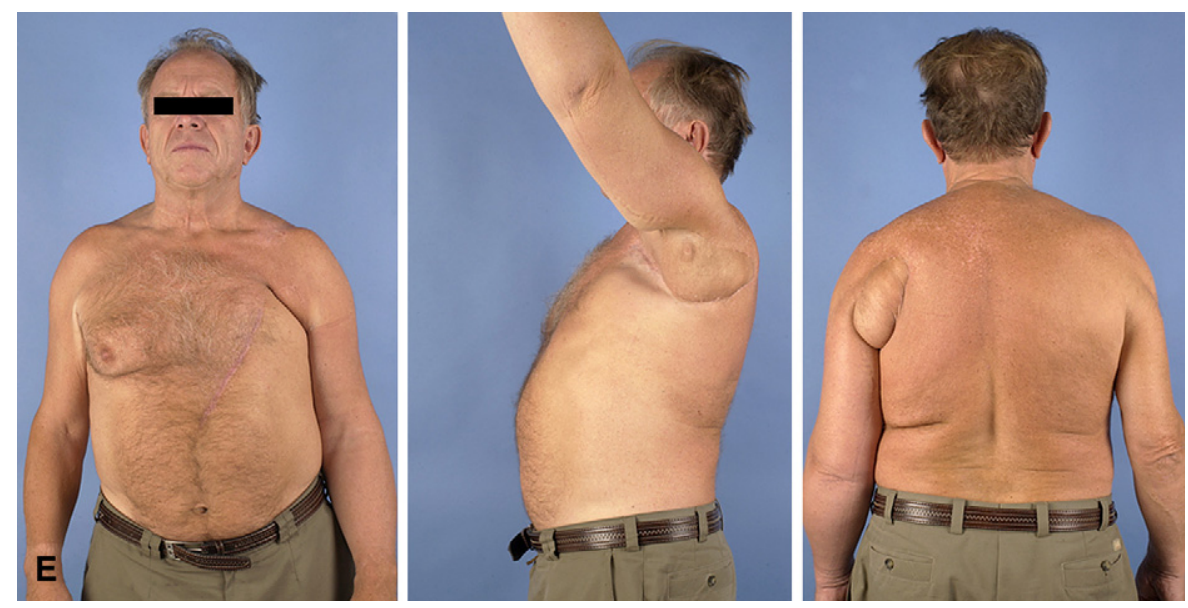

Figure E3E. Patient 3: Result after 18 months. 